2015-10

The oculomotor resonance effect in spatialnumerical mapping

Myachykov, Andriy

http://hdl.handle.net/10026.1/4803

10.1016/j.actpsy.2015.09.006

Acta Psychologica

Elsevier BV

All content in PEARL is protected by copyright law. Author manuscripts are made available in accordance with publisher policies. Please cite only the published version using the details provided on the item record or document. In the absence of an open licence (e.g. Creative Commons), permissions for further reuse of content should be sought from the publisher or author. 
Preprint: $\quad$ Myachykov A., Cangelosi A., Ellis R., Fischer M.H. (2015). The oculomotor resonance effect in spatial-numerical mapping. Acta Psychologica, 161, $162-169$

1

The oculomotor resonance effect in spatial-numerical mapping

Andriy Myachykov, Angelo Cangelosi, Rob Ellis, and Martin H. Fischer

Author note

Andriy Myachykov, Department of Psychology, Northumbria University,

Newcastle-upon-Tyne. Centre for Cognition and Decision Making, National Research

University Higher School of Economics, Russian Federation.

Angelo Cangelosi, School of Computing and Mathematics, University of

Plymouth.

Rob Ellis, School of Psychology, University of Plymouth

Martin H. Fischer, Division of Cognitive Science, University of Potsdam.

This research was supported by the EPSRC grant EP/F026471.

Correspondence concerning this article should be addressed to Andriy

Myachykov, Department of Psychology, Northumbria University, Northumberland

Building, Newcastle upon Tyne, NE1 8ST, United Kingdom, Tel.: +44-191-227-31-

58, Fax: +44-191-227-45-15, e-mail: andriy.myachykov@northumbria.ac.uk

Word count: 5675 words. 


\begin{abstract}
We investigated automatic Spatial-Numerical Association of Response Codes (SNARC) effect in auditory number processing. Two experiments continually measured spatial characteristics of ocular drift at central fixation during and after auditory number presentation. Consistent with the notion of a spatially oriented mental number line, we found spontaneous magnitude-dependent gaze adjustments, both with and without a concurrent saccadic task. This fixation adjustment (1) had a small-number/left-lateralized bias and (2) it was biphasic as it emerged for a short time around the point of lexical access and it received later robust representation around following number onset. This pattern suggests a two-step mechanism of sensorimotor mapping between numbers and space - a first-pass bottom-up activation followed by a top-down and more robust horizontal SNARC. Our results inform theories of number processing as well as simulation-based approaches to cognition by identifying the characteristics of an oculomotor resonance phenomenon.
\end{abstract}

(142 words)

Keywords: attention, embodied cognition, eye movements, oculomotor resonance, ocular drift, SNARC. 


\section{The oculomotor resonance effect in spatial-numerical mapping}

Numbers have long been thought to represent a prototypical abstract knowledge domain. However, the discovery of consistent mappings between numbers and space (Dehaene, Bossini, \& Giraux, 1993) opened the view on systematic sensory and motor biases associated with number concepts. Several reports have now documented a sensorimotor component in number representation known as spatialnumerical association of response codes (SNARC) effect: small numbers, such as 1 or 2, are classified faster with left lateral responses, and larger numbers, such as 8 or 9 , are classified faster with right lateral responses. This effect was demonstrated in various tasks and with different effector systems, including hand, foot, eye, and head movements (for a meta-analysis, see Wood, Nuerk, Willmes, \& Fischer, 2008) and has led to the hypothesis of a spatially oriented Mental Number Line (MNL) as the typical representation of number meaning in our minds. The SNARC effect can be induced both with visually presented numbers and with auditory numbers (Nuerk, Wood, \& Willmes, 2005) consistent with the notion that SNARC reflects a supramodal representation of number concepts. Importantly, Fischer, Castel, Dodd, \& Pratt(2003) demonstrated that visual targets are detected faster in the right visual field if their presentation is preceded by large numbers and they are detected faster in the left visual field when their presentation is preceded by small numbers at the fixation point. More recent studies (Cai \& Li, 2015; Di Bono \& Zorzi, 2013) found a smallnumber advantage in the organization of the horizontally oriented MNL. A similar small-number bias was found for non-symbolic numerosities as well (Lee, Chun, Cho, \& Chung-Ang, 2015). Interestingly, developmental changes in the organization of sensorimotor mappings in number representations confirm that, while a large-number advantage is common in 8-11 year-olds, this advantage shift toward smaller numbers 
in adults (Towse, Loetscher, \& Brugger, 2014). It is possible that this small-number advantage emerged as a result of leftward attentional bias in animals (e.g., Rugani, Vallortigara, Priftis, \& Regolin, 2015) and the fact that small numbers are easier to process overall (Trick \& Pylyshyn, 1994). In addition, the ability of numbers to orient spatial attention was shown to facilitate both manual (e.g., Ristic, Wright, \& Kingstone, 2006) and vocal (Kramer, Stoianov, Umilta, \& Zorzi, 2011; Stoianov Kramer, Umilta, \& Zorzi, 2008) responses to lateral visual targets (see also Galfano, Rusconi, \& Umilta, 2006).

The pervasive and automatic nature of spatial-numerical mappings is not only evident in relatively late manual responses; they are already reflected in early oculomotor shifts that accompany attentional orienting (Hoffman, 1998). For example, Fischer, Warlop, Hill, \& Fias (2004) demonstrated that lateral gazes are initiated faster to the left side after looking at a small number and faster to the right side after looking at a large number. Furthermore, a study by Loetscher, Bockisch, \& Brugger (2008) showed that participants' behaviour in a line bisection task is accompanied with consistent leftward and rightward eye movements dependent on whether the numbers were presented in ascending (e.g., 2-6) or descending (e.g., 6-2) order. Another study (Loetscher, Bockisch, Nicholls, \& Brugger 2010) demonstrated that eye position can predict the forthcoming number in a random number generation task. Conversely, number magnitude affects gaze direction in a free choice task; that is, participants are more likely to choose to look at the right lateral target after fixating small numbers and at the right lateral target after fixating large numbers (Ruiz Fernandez, Rahona, Hervas, Vasquez, \& Ulrich, 2011). Consistent with these results, recent neuroimaging studies yielded direct evidence for a neuroanatomical link between number representations and oculomotor control: Knops, Thirion, Hubbard, 
Michel, \& Dehaene (2009) found partially overlapping parietal areas of activation for leftward saccades and subtraction and similarly for rightward saccades and addition.

It is important to note that spatial-numerical mapping does not exclusively rely upon a horizontally oriented MNL; it also involves a vertical mapping with larger numbers associated with upward and smaller numbers with downward space (Schwarz \& Keus, 2004; Lachmair, Dudschig, de la Vega, \& Kaup, 2014; Viarouge, Hubbard, \& Dehaene, 2014; Winter, Marghetis, \& Matlock, 2015). At the same time, the horizontal dimension seems to have a relatively stronger representation in the "mental number space" than the vertical one (Holmes \& Lourenco, 2012). Also, vertical bias was recently shown to be more evident in mental arithmetic tasks (subtraction/addition) while magnitude-related tasks lead to a stronger horizontal bias (Hartmann, Mast, \& Fischer, 2015).

Put together, these findings suggest that understanding numerical magnitudes involves non-arbitrary spatial orienting and, at least in its horizontal representation, is relatively automatic as it is present in oculomotor responses. However, a number of specific questions related to the exact nature of attentional displacement resulting from number processing remain unexplored. For example, it is not easy to delineate early components of SNARC activation in behavioural studies because the typical performance measure is the latency of discrete responses, a measure that accumulates the time costs of all preceding encoding, decision making, post-decision control, strategic, and motor planning processes. Investigations of eye-movement signatures of SNARC avoid this problem by providing evidence from a behavioural domain which is more automatic and implicit than manual responses. However, all previous oculomotor studies analysed a relatively late parameter of eye movement control, namely saccadic latencies. We reasoned that one could expect to register SNARC 
responses in earlier behavioural and neuropsychological responses. Existing evidence that motivates this expectation can be derived from studies on ultra-rapid linguistic processes that demonstrated extremely early (under 150 milliseconds following input) brain sensitivity to various aspects of linguistic and conceptual semantics (e.g., Moseley, Pulvermuller, \& Shtyrov, 2013; Crouzet, Joubert, Thorpe, \& Fabre-Thorpe, 2012). It is equally possible that processing number words is not dissimilar in that known sensorimotor signatures of conceptual access may reveal their presence in time windows preceding already reported motor and oculomotor responses.

Although this study is the first attempt to analyse the emergence of the SNARC effect in early stationary eye movements, there is more corroborating evidence that provides us with strong theoretical motivation. Stationary eye movements, including ocular tremor, drift, and microsaccades are traditionally thought of as low-level features of fixation maintenance largely encapsulated from higher-level cognitive processes (Martinez-Conde, Macknik, \& Hubel, 2004; Yarbus, 1967). However, several recent studies have shown that fixational eye movements are sensitive to various aspects of higher-level cognitive processes. Microsaccadic behaviour is modulated by both exogenous and endogenous attentional shifts (Betta, Galfano, \& Turatto, 2007; Hafed \& Clark, 2002; Kashihara, Okanoya, \& Kawai, 2014; Laubrock, Engbert \& Kliegl, 2005; Laubrock, Kliegl, Rolfs, \& Engbert, 2010). Changes in microsaccade rate, magnitude, and direction are related to visual search (Kagan \& Hafed, 2013; McCamy, Otero-Millan, Di Stasi, Macknik, \& MartinezConde, 2014) and task difficulty during performance in non-visual tasks (Siegenthaler, Costela, McCamy, Di Stasi, Otero-Millan, Sonderegger, Groner, Macknik, \& Martinez-Conde, 2014). Directionality and the time-course of the ocular drift also reflect information retrieval from memory (Roberts, Wallis, \& Breakspear, 
2013). Finally, studies using the visual world paradigm, where participants see a visual scene while listening to a verbal scene description have shown that participants' eyes anticipate verbal arguments by looking at their semantically related referent before it is mentioned (for review, see Altmann \& Kamide, 2007). This work provides strong support for a rapid and predictive incremental process of concept activation. Based on this work we hypothesized that, as perceivers process numerical magnitudes while maintaining stationary fixations, the directionality of the ocular drift and the accompanying microsaccades may reflect the magnitude of the perceived numbers. As a result, we expected to observe ocular drift and microsaccades to the right following presentation of the larger and to the left following presentation of the smaller numbers.

Finally, our research is also motivated by an interest in delineating the time course of (oculo-) motor activation resulting from SNARC. The study by Fischer et al. (2003) showed facilitation induced by the number-specific attentional shifts only around $700 \mathrm{~ms}$ after digit onset, thus suggesting a relatively slow time-course of the number-space mapping mechanism (Fischer et al., 2003). Other studies using manual responses (e.g., Casarotti, Michielin, Zorzi, \& Umiltà, 2007; Dodd, Van der Stigchel, Adil Leghari, Fung, \& Kingstone, 2008; Ristic, Wright, \& Kingstone, 2006) replicated and extended this finding and converged on a comparable time course. It is also well established that the SNARC effect is stronger for slower than for faster responses (Gevers, Verguts, Reynvoet, Caessens, \& Fias, 2006). However, relatively large inertial forces must be overcome in manual-response tasks, thus leading to relatively slow response times. This biomechanical contamination, as well as the accrual of time costs from all cognitive stages prior to response execution, may overestimate the minimal time needed for number meaning to affect motor output. 
Oculomotor behaviour does not have to overcome large inertial forces and can be initiated much faster than manual behaviour. Its short latency also implies that some of the cognitive processes contaminating manual responses may be absent, at least in the early parts of the oculomotor latency distribution (cf. Carpenter, 1977). This suggests that oculomotor recording in response to numbers can be a more sensitive readout of the time course of conceptual activation and its associated motor resonance.

Below, we report two experiments that used a method similar to the visual world methodology: Participants' task was to classify auditorily presented numbers in a go-nogo task and to look at lateralized visual probes. Their eye position was analysed in a two-dimensional plane in relation to their real-time apprehension of number magnitude. We investigated how this number apprehension affected displacement of overt visual attention by analysing gradual changes in eye position both with (Experiment 1) and without (Experiment 2) a concurrent saccadic task. The results provide evidence for an automatic oculomotor resonance effect both during saccade preparation and when no such preparation is necessary.

\section{Method}

The main purpose of both experiments was to investigate how the relative magnitude of auditorily perceived numbers activates spatial attentional mechanisms. Participants' covert attention deployment was inferred from their saccadic latencies to the visually presented probes. We used eye position prior to the saccade as an indicator of overt attention allocation to track the time course of number-space mappings. 


\section{Participants}

Self-reportedly right-handed native speakers of English participated in both studies. There were 19 participants ( 2 males) in Experiment 1 and 17 participants (2 males) in Experiment 2. Average age of participants in Experiment 1 was 19.8 years; average age in Experiment 2 was 22 years. All participants had normal or correctedto-normal vision. Each participant's eye dominance was determined using a procedure similar to the one described in Roth, Lora, and Heilman (2002): Participants were run on variants of the Porta test, the Miles test, and the convergence near-point test. Participants who performed as right-eye dominant on two out of the three tests were classified as right-eye dominant; participants who performed as left-eye dominant on two out of the three tests were classified as left-eye dominant. Prior to the experiment, participants' handedness was formally assessed by administering a modified version of Annett's handedness questionnaire (Annett, 1970). This assessment confirmed that participants in both studies were predominantly right-handed (scores in Experiment 1 between 34 and 36, mean of 35.3; scores in Experiment 2 between 33 and 36, mean 33.7). Participants either received course credit or $£ 6$ for their participation.

\section{Materials and Design}

In both experiments, we used the auditory numbers $1,3,5,7$, and 9. Only odd numbers were used to control the MARC effect, according to which odd and even numbers are associated with left and right space, respectively (e.g., Nuerk et al., 2005). The number 5 was used to ensure that participants constantly attended to the magnitude of the presented number names: we instructed them to signal the detection of number 5 by pressing a button. These "catch" trials constituted $20 \%$ of the total number of trials in both studies. In Experiment 1, we used a $2 \times 2 \times 3$ factorial design with the following independent variables: Number Magnitude (Small: 1, 3 vs. Large: 
7, 9) of the auditory number cue, Visual Probe Location (left vs. right visual field), and Probe Onset Latency (POL) (400 ms, $800 \mathrm{~ms}$, and 1,200 ms from offset of the number word). In Experiment 2, we used a task that required continuous eye fixation without any subsequent spatial task (although participants still had to indicate by key press when number 5 was played). The main purpose of this second experiment was to determine whether the oculomotor resonance effect discovered in the first Experiment resulted from the necessity to launch a saccade and fixate a lateral visual probe or whether it would be observed in passive number processing as well. Hence, in Experiment 2, we manipulated only one independent variable: the numerical magnitude of the number word (Small: 1, 3; vs. Large: 7, 9; catch trials with magnitude 5 required a button response).

Auditory materials consisted of five audio (.wav) files of the number names spoken by a male speaker of English and recorded in a sound-attenuated laboratory setting. All audio files were of $1000 \mathrm{~ms}$ length. Visual materials were presented on a 1024x768-pixel white screen with a solid black circle in the center. The circle's diameter was 20 pixels. Visual probes in Experiment 1 were solid red circles with 30 pixels in diameter. The left probe appeared centred on the coordinates $256 \times 384$ pixels, equidistant from the left edge of the screen and its central point. Correspondingly, the right probe was centred on coordinates $768 \times 384$ pixels, equidistant from the right edge of the screen and the central fixation point. This corresponds to probe eccentricities of approximately 3 degrees. The same auditory materials were used in Experiment 2.

\section{Apparatus}

Both experiments were implemented in SR-Research Experiment Builder software version 1.5.201 (SR Research, 2009). An Eye-Link 1000 desk-mounted eye 
tracker monitored participants' eye movements with $1000 \mathrm{~Hz}$ sampling rate. We recorded with high spatial accuracy (less than 0.5 degrees of visual angle, see http://www.sr-research.com/EL 1000.html) and further enhanced spatial precision by using a head-and-chin rest and aggregating across successive samples (see below). The experimental materials were presented on a 19' ViewSonic G90fB monitor of a DELL Optiplex 755 desktop computer running at a display refresh rate of 90 Hertz. The eye-tracking data were extracted and filtered using SR-Research Data Viewer Version 1.91 (SR Research, 2009). A custom-developed Python script (SR Research, 2009) was used to extract time-series data from the participants' sample reports (see below). Participants signalled catch trials by pressing the right shooting key on a Microsoft Sidewinder game-pad integrated with the Eye-Link eye-tracking system.

\section{Procedure}

After giving informed consent, which was prepared conforming to the Declaration of Helsinki, the participant sat at a distance of $60 \mathrm{~cm}$ centrally in front of the monitor. Viewing was binocular but only the dominant eye was tracked. Before the main experimental session, each participant received ten practice trials with 500 ms POL, hearing each number twice. There was no recording of the participants' eye movements during the practice session. Prior to the experimental session, the eyetracking equipment was calibrated to a 9-point calibration screen. A desk-mounted head-and-chin rest restricted the participant's head movements.

During the experimental session, each participant received an individually pseudo-randomized sequence of 240 experimental trials (192 target trials and 48 catch trials). Figure 1 illustrates a typical target trial sequence in Experiment 1.

(Figure 1 here) 
Each experimental trial started with the presentation of the central fixation screen. The onset of the audio file was gaze-contingent to its presentation: The participant had to fixate the central fixation point for a minimum of $150 \mathrm{~ms}$ for the auditory number to be played. The participant then heard the number's name binaurally via headphones and indicated as soon as possible by pressing the right shooting key on the game pad when the presented number was 5 . The central fixation point disappeared together with the number name onset in order to disengage oculomotor fixation circuits, thus allowing more freedom for oculomotor shifts and ensuring more rapid saccade initiation (the "gap effect"; e.g., Fischer \& Weber, 1993). We hypothesized that this attentional disengagement prior to number processing should facilitate the magnitude-related spatial bias.

Only the right shooting key was used to indicate number recognition because all participants were right-handed. We ensured that participants were not alerted to catch trials by presenting visual probes during both experimental and catch trials. There was a POL of 400,800 , or $1200 \mathrm{~ms}$ between the offset of the auditory number file and the onset of the visual attention probe (the red circle) that appeared unpredictably on the left or right side of the central fixation. The offset of the visual probe was saccade-contingent: Participants had to fixate in the $100 \times 100$-pixel rectangular area of the screen around the probe. After detecting a successful fixation on the probe the central fixation screen appeared again and the next trial followed.

Participants were told that the sole purpose of the study was to investigate how quickly people can recognize numbers' identities. The experimental instruction to participants was to fixate the central fixation point, continue looking at the point on the screen when the fixation point disappears, listen to the number's name played in the headphones, fixate the red dot as soon as it appeared on the screen, and press the 
response key as quickly as possible when the number 5 is detected. A debriefing session at the end of each experimental session established that the true purpose of the study had remained unknown to all participants.

In Experiment 2, we followed a similar procedure with the exception that there was no visual probe that would require saccadic eye movements. During the experimental session, each participant received an individually pseudo-randomized sequence of 40 trials (32 target trials and 8 catch trials). Each trial started with the presentation of the central fixation screen. This screen was replaced with a blank screen once the participant had successfully fixated the central fixation dot for 150 ms. The onset of the number's name was simultaneous with the presentation of the blank screen. Eye position was recorded for $2500 \mathrm{~ms}$ (1000 ms of Number Word presentation plus an additional $1500 \mathrm{~ms}$ ). The instruction to all participants was to fixate the central fixation point and to continue looking at the point on the blank screen where the fixation point had previously been shown. Participants were also instructed to press the response key as soon as they heard the auditory number 5 . Debriefing confirmed that participants remained unaware about the purpose of the study.

\section{Results}

First, we assessed error rates in participants' identification of catch trials. Errors were very rare, consistent with the simplicity of the task: Participants indicated the presence of number 5 in $99 \%$ of catch trials (hits) in both studies and made less than $1 \%$ false alarms (button presses in response to other number names).

Eye-tracking data were filtered and exported from the raw EDF files with Data Viewer software (SR Research, 2009). Fixation duration threshold was set at $50 \mathrm{~ms}$ minimum and saccade amplitude threshold was set at $3.0^{\circ}$. Blink-related saccades 
were not included in the output. Two aspects of eye behaviour were of special interest to us. First, we were interested in gradual drift of the eye position either during (Experiment 1) or both during and after (Experiment 2) listening to number names. Second, in Experiment 2 we analysed the parameters of probe-directed saccades as a function of the relative magnitude of the auditory cue in two time intervals. The CueTo-Probe Interval (CPI) covered eye behaviour from the onset of the auditory cue to the onset of the visual probe in Experiment 1 and the Probe-To-Response Interval (PRI) covered the time period from the onset of the visual probe to the completion of the probe-directed saccade. In Experiment 2, we recorded continuously the fixation position relative to the central fixation for the duration of each trial.

\section{Ocular drift analysis}

For the purposes of ocular drift analyses we created a time-series bin report with the help of a custom-made Python script (SR Research, 2009). Individual average fixation positions were available for each millisecond as a function of trial time. The bin report plots mean average $\mathrm{X}$ and $\mathrm{Y}$ gaze coordinates as a moving average with a width of $50 \mathrm{~ms}$. The overall average eye position in Experiment 1 during CPI had horizontal and vertical coordinates of 509 x 386 pixels, respectively. The overall average eye position in Experiment 2 had horizontal and vertical coordinates of $509 \times 388$ pixels, respectively. Figures 2 and 3 represent changes in horizontal eye position in our two experiments. Figure 2 illustrates ocular drift in Experiment 1 during and after the uptake of numerical information but before a saccade to the probe. Figure 3 illustrates the average change of the participants' horizontal eye position as a function of trial time for the two experimental conditions (Large vs. Small Numbers).

(Figures 2 and 3 here) 
The diverging lines in both Figures show that the average horizontal eye position shifted to the left in response to small magnitude numbers (dotted line) and to the right in response to large magnitude numbers (solid line) prior to any overt saccadic behaviour, presumably as participants associated the numerical magnitude with its direction. This impression was confirmed with statistical testing, where all effects with corresponding $p$-values below the conventional cut-off of $p=.05$ were significant. Benjamini-Hochberg False Discovery Rate correction (Bejamini \& Hochberg, 1995) was applied for multiple comparisons in both studies. Hence, t-test threshold of $+/-2.11$ was used. Paired-samples t-tests were performed on every $50 \mathrm{~ms}$ bin in both studies.

Our analysis confirmed that the SNARC effect in Experiment 1 first emerged briefly $200 \mathrm{~ms}$ after number word onset $(t(18)=-2.34)$; it remained significant until $400 \mathrm{~ms}$ after number word onset. The effect was firmly established again around 700 ms after number word offset $(t(18)=-2.19)$ this time lasting until the end of the cue to probe interval. In Experiment 2 the same magnitude-related horizontal ocular drift was first registered $850 \mathrm{~ms}$ after number word onset $(t(16)=-2.33)$. It was again short-lived, lasting for $200 \mathrm{~ms}$ and disappearing shortly after the number word offset. The effect re-emerged $600 \mathrm{~ms}$ after the number word offset $(t(16)=-2.14)$ lasting now until the end of the tested time period.

We also analyzed ocular drift along the vertical axis taking average $\mathrm{Y}$ coordinates as the dependent variable. However, these analyses did not return reliable results. We discuss potential reasons for the relative dominance of the horizontal ocular drift further below. 


\section{Saccade analysis}

In order to examine participants' saccadic behaviour in Experiment 1, we created a Saccade Report for PRI with the following dependent variables: (1) Saccade Launch Time (time from visual probe onset to saccade onset) and (2) Saccade Launch X Coordinate. These data were trimmed to fall within two standard deviations around individual participants' means, leaving us with $89-93 \%$ of the total data, depending on the dependent variable in question. Importantly, we removed all eye position data reflecting actual saccade executions; the procedure led to roughly equal left and right side probe onsets contributing to the positional means we report below. The three data sets were each entered into a $2 \times 2 \times 3$ factorial analysis of variance (ANOVA) with the independent factors of Numerical Magnitude (Small: 1, 3 vs. Large: 7, 9), Visual Probe Location (Left vs. Right), and POL (400, 800, and 1,200 ms from cue offset).

Analysis of the Saccade Launch Onset Latencies in PRI revealed a main effect of POL $(F(2,36)=3.50)$ with a reliable quadratic trend $(F(1,18)=8.90)$. Post-hoc pair-wise comparisons confirmed the presence of a U-shaped fore-period effect comparable to the one reported in Fischer et al. (2003): Overall, participants were slower to initiate probe-directed saccades after a $400 \mathrm{~ms}$ delay (mean $=168 \mathrm{~ms}$ ) than after an $800 \mathrm{~ms}$ delay $($ mean $=160 \mathrm{~ms})(t(18)=2.88)$. The expected interaction between probe location and number magnitude failed to reach significance, $F(1,18)=$ $.066, \mathrm{p}=.80$. There was, however, a reliable interaction between Numerical Magnitude and POL $(F(2,36)=3.80)($ see Figure 4$)$.

(Figure 4 here)

Examining this interaction revealed a reliable difference between Small and Large Magnitude trials only in the $400 \mathrm{~ms}$ POL condition (165 ms and $170 \mathrm{~ms}$, respectively $(t(18)=1.924)$. Thus, saccadic responses to probes on either side were 
initiated faster following small numbers at short (400 ms) POL intervals and also following large numbers at medium $(800 \mathrm{~ms})$ POL intervals.

\section{Discussion}

Extending previous work on spatial-numerical associations, we found a novel and systematic bias in involuntary eye drift and the subsequent adjustment of the saccade launch site: Following a small auditory number ( 1 or 3 ) eye position drifted to the left, and following a large auditory number ( 7 or 9 ) eye it drifted to the right. A spontaneous and automatic gaze adjustment following auditory numbers was present in both studies. This reliable and novel finding is consistent with the representation of numerical magnitudes along a horizontally oriented MNL. Importantly, this spatial bias, albeit very short-lived, was already present during number word presentation, thus further reinforcing the idea that spatial-numerical mappings can be rapid and automatic with their presence detectable already during early stages of lexical access. This result suggests an automatic and early covert attentional shift resulting from number processing as soon as enough minimal semantic information is available for the spatial-numerical mapping to be activated. As noted above, such rapid effects in language comprehension and language learning were registered before (e.g., Moseley

et al., 2013). However, to the best of our knowledge we provide the first report of relatively rapid activation of sensorimotor mappings and associated attentional shifts for number processing.

Following its early and short-lived shift manifestation, the spatial-numerical association was firmly re-established later after the auditory number presentation. The fact that this bias was induced automatically and prior to probe onset in the form of involuntary gaze shifts confirms the obligatory nature of horizontal spatial-numerical mappings. Importantly, while the magnitude-related ocular drift in Experiment 1 
could, in principle, be a result of saccade preparation processes (e.g., participants trying to "predict" where the probe would be displayed), this strategic planning account is refuted by the data from Experiment 2, demonstrating that the drift effect also occurred even when no response (except for the occasional catch trials) was necessary. Hence, the observed magnitude-related horizontal drift with and without a saccadic task extends previously reported results of spatial congruency between activated meaning and ongoing behaviour during language processing (e.g., Glenberg \& Kaschak, 2002; Zwaan \& Taylor, 2006), where the notion of motor resonance is used to capture this effect. We therefore call this analogous behaviour in our task an oculomotor resonance effect. Similarly to the motor resonance effect in language comprehension, the oculomotor resonance effect reflects how processing domainspecific information (e.g., magnitude meaning or verb meaning) results in corresponding changes in domain-general processing (e.g., motor simulation and corresponding changes in overt behaviour). Therefore, both effects may be understood as embodied signatures of symbol comprehension (Barsalou, 2008; Pulvermüller, 2013).

Our analysis of the underlying dynamics effectively reflects a biphasic SNARC effect: An automatic, first-pass bottom-up activation from early lexical access is followed by a later but more robust top-down spatial SNARC. This biphasic activation of SNARC is a novel finding; as such, it requires further investigation. This is an intriguing aspect of the data but it was replicated and there are theoretical ideas to justify it. For example, it seems plausible to invoke classical two-process models of numerical cognition (e.g., Banks \& Flora, 1977), which postulate a rapid global and a subsequent more refined assessment of number magnitudes. Although the task in the present experiments did not explicitly require such analysis, it may occur 
automatically when evaluating numbers (with regard to the go-no-go criterion). Importantly, further studies with our paradigm allow for further test of such biphasic activation of conceptual information in other linguistic knowledge domains, for example, spatial language, words denoting valence, and words with temporal semantics. Put together, our analysis of ocular drift in both studies suggests that understanding of numerical magnitude information relies on a mapping between the position of this number on the MNL via an attentional orienting mechanism that affects the response the participants are currently preparing (i.e., the horizontally oriented saccade).

One peculiar aspect of the observed ocular drift in Experiment 2 is that a shift to left for the small numbers shortly before number offset is unaccompanied by a similar bias for the large numbers. In contrast, circa $600 \mathrm{~ms}$ after number offset a more pronounced shift to the right is observed. This pattern provides more detail to the notion of stepwise activation of the number-induced spatial mapping. In line with the proposed global evaluation of the size code (Banks \& Flora, 1977) it is not surprising that there is an early left/small number bias and a later right/large number bias. A similar attentional preference for small numbers during activation of SNARC was recently documented by Cai and Li (2015), whose participants detected visual targets after their attention was cued by means of a small or a large number preview. The data clearly demonstrated that small numbers had an advantage over large numbers in capturing attention. Furthermore, recent research by Lee and colleagues (Lee et al., 2015) revealed a similar left-side bias in processing non-symbolic numerosities. The authors' interpretation of this leftward attentional bias is based on "pseudo-neglect" for the right side of space, resulting in underestimation of numerosities presented on the right side. Finally, research on the developmental 
changes in the organization of sensorimotor mappings in number representations confirms that, while a large-number advantage is common in 8-11 year-olds, this advantage shifts toward smaller numbers in adults (Towse, Loetscher, Brugger, 2014). Another aspect of the data pattern that requires a special note is the absence of an interaction between magnitude and probe location. One could expect that both early effects in ocular drift would correlate with the parameters of the subsequent saccades, meaning that if a small number led to an adjustment of the eye position to the left then microsaccades to the left should also be faster. We did, however, not find such effects on saccadic latencies. However, the two processes (ocular drift vs. saccade planning and execution) may be dissociated. Such dissociations of components are not uncommon, for example, dissociations of various signatures of attention deployment, such as EEG signatures without accompanying behavioural correlates (Sallilas, El Yagoubi, \& Semenza, 2008; Schuller, Hoffmann, Goffaux, \& Schiltz, 2015). Furthermore, saccades and ocular drift differentially modulate neuronal activity (Kagan, Gur, \& Snodderly, 2008).

A similar dissociation can be found in studies of manual affordances: While neurophysiological studies often reveal involvement of the attentional system during activation of affordance effects (di Pelegrino, Rafal, Tipper, 2005; Handy, Grafton, Shroff, Ketay, Gazzaniga, 2003; Handy, Borg, Turk, Tipper, Grafton, Bazzaniga, 2005) behavioural studies typically fail to find similar interactions between attention and manipulability (e.g., Hommel, 1993; Phillips \& Ward, 2002; Vainio, Ellis, \& Tucker, 2007). Hence, while our attentional system seems to be involved in the early apprehension of manipulable objects, this activation is not always accompanied by a corresponding latency advantage in overt responses. 
Although numerous reports document the presence a vertical SNARC in spatial-numerical mappings, the horizontal shift that we showed in our studies was not accompanied by a reliable vertical displacement. However, vertical signatures of SNARC have thus far been found in overt response tasks (e.g. Holmes \& Lourenco, 2012; Lachmair et al., 2014; Viarouge et al., 2014; Wiemers et al., 2014; Winter \& Matlock, 2013). It is quite possible that horizontal and vertical mapping dimensions in number processing are not activated simultaneously and that the horizontal space is available for mapping earlier and in a more automatic fashion. Confirming this logic, some studies have found that the horizontal dimension is indeed more prevalent during number processing, at least in adults (Holmes \& Lourenco, 2012). This asymmetric prevalence was fortified by our use of horizontal and no vertical probes. Also, a recent eye movement study showed that the vertical shift may be more evident in mental arithmetic (subtraction/addition) while magnitude tasks show a stronger reliance on horizontal mappings (Hartmann, Mast, \& Fischer, 2015).

Finally, the time course of SNARC in the present paradigm differed from previous results and was also task-dependent: In the saccadic task, number magnitude started to affect horizontal eye position already during auditory number presentation, reliably predicting the positional bias of the target saccade launch site. In Experiment 2, where no saccadic response was made, this horizontal drift appeared briefly during number apprehension and reinstated itself once the number name was fully presented. Given that SNARC is already evident in reaction times of around half a second in many button pressing tasks, why did the mapping effect on eye position emerge so late, requiring several hundred milliseconds after number presentation to emerge? First, it is worth remembering that this result is consistent with previous work on slow-emerging attention deployment in response to uninformative and task-irrelevant 
numbers (Dodd, Van der Stigchel, Adil Leghari, Fung, \& Kingstone, 2008; Fischer et al., 2003), although the present experiments employed catch trials to ensure number processing. More importantly, auditory presentation of numerical information may be associated with a slower mapping function than visual presentation, which involves participants already attending to visual space (cf. Beauchamp, Lee, Argall, \& Martin, 2004). In other words, the auditory input might only be mapped onto space once a modality switch has been performed. Related to this, the early fixation drifts we observed in both experiments, although not reliable, might correspond to the typical SNARC pattern that associates the magnitude meaning of the numbers with space, yet it failed to reach significance here because of the delayed modality switch. Thus, two processing steps may be involved in the oculomotor resonance effect. This proposal requires further testing.

In summary, the current paper documents an obligatory mapping of number magnitude onto space via orienting of attention along the mental number line. The observed oculomotor biases demonstrate an oculomotor resonance effect in number processing that indicates a motor simulation as part of number comprehension. 


\section{Acknowledgments}

This research was supported by the EPSRC grant: Vision, Action, and Language Unified by Embodiment (EP/F026471) awarded to Rob Ellis, Angelo Cangelosi, and Martin H. Fischer. 


\section{References}

Altmann, G.T.M., \& Kamide, Y. (2007). The real-time mediation of visual attention by language and world knowledge: Linking anticipatory (and other) eye movements to linguistic processing. Journal of Memory and Language, 57(4), $502-518$.

Andres, M., Olivier, E., \& Badetsd, A. (2008). Actions, words, and numbers: A motor contribution to semantic processing? Current Directions in Psychological Science, $17(5), 313-317$.

Annett, M. (1970). A classification of hand preference by association analysis. British Journal of Psychology, 61, 303-321.

Banks, W.P. \& Flora, J. (1977). Semantic and perceptual processes in symbolic comparisons. Journal of Experimental Psychology, 3(2), 278-290.

Barsalou, L.W. (2008). Grounded cognition. Annual Review of Psychology, 59, 617645.

Beauchamp, M., Lee, K., Argall, B., \& Martin, A. (2004). Integration of auditory and visual information about objects in superior temporal sulcus. Neuron, 41, 809-823.

Betta, E., Galfano, G., \& Turatto, M. (2007). Microsaccadic response during inhibition of return in a target-target paradigm. Vision Research, 47, 428-436

Bibi, R. \& Edelman, J.A. (2009). The influence of motor training on human express saccade production. Journal of Neurophysiology, 102, 3101-3110.

Brunamonti, E., Falcone, R., Genovesio, A., Costa, S., \& Ferraina, S. (2012). Gaze orientation interferes with mental numerical representation. Cognitive Processing, 13, 375-379.

Cai, Y-C. \& Li, S-X. (2015). Small number preference in guiding attention. Experimental Brain Research, 233, 539-550. 
Cappelletti M., Freeman E.D., \& Cipolotti, L. (2009). Dissociations and interactions between time, numerosity, and space processing. Neuropsychologia, 47, 27322748.

Carpenter, R.H.S. (1977). Movements of the Eyes. Pion: London.

Carpenter, R.H.S. (1981). Oculomotor Procrastination, In Eye Movements: Cognition and Visual Perception, D. F. Fisher, R. A. Monty, \& J. W. Senders (Eds), (pp. 237-246). Hillsdale: Lawrence Erlbaum.

Casarotti, M., Michielin, M., Zorzi, M., \& Umiltà, C. (2007). Temporal order judgment reveals how number magnitude affects visuospatial attention. Cognition, $102,101-117$.

Casasanto, D. \& Boroditsky, L. (2008). Time in the Mind: Using space to think about time. Cognition, 106, 579-593.

Crouzet, S.M., Joubert, O, Thorpe, S.J., \& Fabre-Thorpe, M. (2012). Animal detection precedes access to scene category. PLoS ONE, 7(12), e51471.

Daar, M., \& Pratt, J. (2008). Digits affect actions: The SNARC effect and response selection. Cortex, 44, 400-405.

Dehaene, S., Bossini, S., \& Giraux, P. (1993). The mental representation of parity and number magnitude. Journal of Experimental Psychology: General, 122, 371-396.

Di Luca, S., Pesenti, M., Vallar, G., \& Girelli, L. (2013). Numbers reorient visuospatial attention during cancellation tasks. Experimental Brain Research, 225, 549557.

Di Bono, M.G. \& Zorzi, M. (2013). The spatial representation of numerical and non-numerical ordered sequences: Insights from a random generation task. The Quarterly Journal of Experimental Psychology, 66(12), 2348-2362. 
di Pelegrino, G., Rafal, R. Tipper, S.P. (2005). Implicitly Evoked Actions Modulate Visual

Selection: Evidence from Parietal Extinction. Current Biology, 15, 1469-1472.

Dodd, M.D., Van der Stigchel, S., Adil Leghari, M., Fung, G., \& Kingstone, A. (2008). Attentional SNARC: There's something special about numbers (let us count the ways). Cognition, 108, 810-818.

Fischer, B. \& Ramsperger, E. (1984). Human express saccades: extremely short reaction times of goal directed eye movements. Experimental Brain Research, 57, 191-195.

Fischer, B. \& Weber, H. (1993). Express saccades and visual attention. Behavioral and Brain Sciences, 16, 553-610.

Fischer, M.H. (2008). Finger counting habits modulate spatial-numerical associations. Cortex, 44, 386-392.

Fischer, M.H., Castel, A.D., Dodd, M.D., \& Pratt, J. (2003). Perceiving numbers causes spatial shifts of attention. Nature Neuroscience, 6, 555-556.

Fischer, M.H., Warlop, N., Hill, R.L., \& Fias, W. (2004). Oculomotor bias induced by number perception. Experimental Psychology, 51(2), 1-7.

Fischer, M.H., Shaki, S., \& Cruise, A. (2009). It takes only one word to quash the SNARC. Experimental Psychology, 56(5), 361-366.

Fischer, M. H., \& Zwaan, R. A. (2008). Embodied language - A review of the role of the motor system in language comprehension. Quarterly Journal of Experimental Psychology, 61(6), $825-850$.

Foroni, F. \& Semin, G.R. (2009). Language that puts you in touch with your bodily feelings: The multimodal responsiveness of affective expressions. Psychological Science, 20(8), 974-980. 
Freeman, J. B., Dale, R., \& Farmer, T. A. (2011). Hand in motion reveals mind in motion. Frontiers in Psychology, Vol. 2, Article 59

Galfano, G., Rusconi, E., \& Umiltà, C. (2006). Number magnitude orients attention, but not against one's will. Psychonomic Bulletin \& Review, 13, 869-874.

Gevers, W., Verguts, T., Reynvoet, B., Caessens, B., \& Fias, W. (2006). Numbers and space: Computational model of the SNARC effect. Journal of Experimental Psychology: Human Perception and Performance, 32, 32-44.

Glenberg, A.M., Sato, M., Cattaneo, L., Riggio, L., Palumbo, D., \& Buccino, G. (2008). Processing abstract language modulates motor system activity. Quarterly Journal of Experimental Psychology, 61(6), 905-919.

Hafed, Z.M. \& Clark, J.J. (2002). Microsaccades as an overt measure of covert attention shifts. Vision Research, 42, 2533-2545.

Handy, T.C., Grafton, S.T., Shroff, N.M., Ketay, S., \& Gazzaniga, M.S. (2003). Implicitly Evoked Actions Modulate Visual Selection: Evidence from Parietal Extinction. Nature Neuroscience, 6(4), 421-427.

Handy, T.C., Borg, J.S., Turk, D.J., Tipper, C.M., Grafton, S.T., Gazzaniga, M.S. (2005). Placing a tool in the spotlight: spatial attention modulates visuomotor responses in cortex. Neuroimage, 26, 266-276.

Hartmann, M., Mast, F.W., \& Fischer, M.H. (2015). Spatial biases during mental arithmetic: Evidence from eye movements on a blank screen. Frontiers in Psychology, 6, 12.

Hoffman, J.E. (1998). Visual attention and eye movements. In H. Pashler (Ed.), Attention. (pp. 119-153). Hove: Psychology Press. 
Holmes, K.J. \& Lourenco, S.F. (2012). Orienting numbers in mental space: Horizontal organization trumps vertical. The Quarterly Journal of Experimental Psychology, 65(6), 1044-1051.

Hommel, B. (1993). The role of attention for the Simon effect. Psychological Research, 55, 208-222.

Ito, Y., \& Hatta, T. (2004). Spatial structure of quantitative representation of numbers: Evidence from the SNARC effect. Memory \& Cognition, 32, 662-673.

Kagan, I., Gur, M., \& Snodderly, D.M. (2008). Saccades and drifts differentially modulate neuronal activity in V1: Effects of retinal image motion, position, and extraretinal influences. Journal of Vision, 8(14), 1-25.

Kagan, I. \& Hafed, Z.M. (2013). Active vision: microsaccades direct the eye to where it matters most. Current Biology, 23, R712-R714.

Kashihara, K., Okanoya, K., \& Kawai, N. (2014). Emotional attention modulates microsaccadic rate and direction. Psychological Research, 78(2), 166-179.

Keus, I.M., \& Schwarz, W. 2005. Searching for the locus of the SNARC effect: Evidence for a response-related origin. Memory and Cognition, 33, 681-695

Knops, A., Thirion, B., Hubbard, E. M., Michel, V., \& Dehaene, S. (2009). Recruitment of an area involved in eye movements during mental arithmetic, Science, 324, 1583-1585.

Kramer, P., Stoianov, I., Umiltà, C., \& Zorzi, M. (2011). Interactions between perceptual and numerical space. Psychonomic Bulletin and Review, 18, 722-728.

Lachmair, M., Dudschig, C., de la Vega, I., \& Kaup, B. (2014). Relating numeric cognition and language processing: Do numbers and words share a common representational platform? Acta Psychologica,148, 107-114. 
Laubrock, J., Engbert, R., \& Kliegl, R. (2005). Microsaccade dynamics during covert attention. Vision Research, 45, 721-730.

Laubrock, J., Kliegl, R., Rolfs, M., \& Engbert, R. (2010). When do microsaccades follow spatial attention. Attention, Perception \& Psychophysics, 72(3), 683-694

Lee, D., Chun, J., Cho, S., \& Chung-Ang (2015). Leftward Perceptual Bias surpasses the SNARC effect in Non-Symbolic Numerosity Comparison Performance. Poster presented at 2015 Cognitive Neuroscience Society meeting, San Francisco, CA.

Loetscher, T., Bockisch, J., \& Brugger, P. (2008). Looking for the answer: the mind's eye in number space. Neuroscience, 151, 725-729.

Loetscher, T., Bockisch, C.J., Nicholls, M.E.R., \& Brugger, P. (2010). Eye position predicts what number you have in mind. Current Biology, 20(6), R264-R265.

Martinez-Conde, S., Macknik, S.L., \& Hubel, D.H. (2004). The role of fixational eye movements in visual perception. Nature Review Neuroscience, 5, 229-240.

McCamy, M.B., Otero-Millan, J., Di Stasi, L.L., Macknik, S.L., \& Martinez-Conde, S. (2014). Highly Informative Natural Scene Regions Increase Microsaccade Production during Visual Scanning. The Journal of Neuroscience, 34(8), 29562966

Moeller, K., Fischer, M. H., Nuerk, H.-C., \& Willmes, K. (2009). Sequential or parallel processing of two-digit numbers? Evidence from eye-tracking. Quarterly Journal of Experimental Psychology, 62(2), 323-334.

Moeller, K., Fischer, M. H., Nuerk, H. C., \& Willmes, K. (2009). Eye fixation behaviour in the number bisection task: Evidence for temporal specificity. Acta Psychologica, 131, 209-220. 
Moseley, R.L., Pulvermuller, F., \& Shtyrov, Y. (2013). Sensorimotor semantics on the spot: brain activity dissociates between conceptual categories within $150 \mathrm{~ms}$. Scientific Reports, 3, 1928.

Nuerk, H-C., Wood, G., \& Willmes, K. (2005). The universal SNARC effect: The association between number magnitude and space is amodal. Experimental Psychology, 52(3), 187-194.

Pecher, D., Boot, I., \& Van Dantzig, S. (2011). Abstract Concepts: Sensory-motor Grounding, Metaphors, and Beyond. In B.H. Ross (Ed.), The Psychology of Learning and Motivation. (pp. 217-248). Academic Press: Burlington.

Phillips, J.C. \& Ward, R. (2002). S-R correspondence effects of irrelevant visual affordance: Time course and specificity of response activation. Visual Cognition, 9(4/5), 540-558.

Pulvermüller, F. (2005). Brain mechanisms linking language and action. Nature Reviews Neuroscience, 6, 576-582.

Ristic, J., Wright, A., \& Kingstone, A. (2006). The number line effect reflects topdown control. Psychonomic Bulletin \& Review, 13(5), 862-868.

Roberts, J.A., Wallis, G., \& Breakspear, M. (2013). Fixational eye movements during viewing of dynamic natural scenes. Frontiers in Psychology, 4,797.

Rugani, R., Vallortigara, G., Priftis, K., \& Regolin, L. (2015). Number-space mapping in the newborn chick resembles humans' mental number line. Science, 347,534536.

Ruiz Fernandez, S. Rahona, J.J., Hervas, G., Vazquez, C., \& Ulrich, R. (2011). Number magnitude determines gaze direction: Spatial-numerical association in a free-choice task. Cortex, 47, 617-620. 
Sallilas, E., El Yagoubi, R., \& Semenza, C. (2008). Sensory and cognitive processes of shifts of spatial attention induced by numbers: an ERP study. Cortex, 44, 406-413.

Schuller, A.-M., Hoffmann, D., Goffaux,V., \& Schiltz, C. (2015). Shifts of spatial attention cued by irrelevant numbers: Electrophysiological evidence from a target discrimination task. Journal of Cognitive Psychology, 27(4), 442-458.

Schwarz, W. \& Keus, I. (2004). Moving the eyes along the mental number line: Comparing SNARC effects with manual and saccadic responses. Perception \& Psychophysics, 66, 651-664.

Shaki, S. \& Fischer, M. H. (2008). Reading space into numbers - A cross-linguistic comparison of the SNARC effect. Cognition, 108(2), 590-599.

Shaki, S., Fischer, M. H., \& Petrusic, W. M. (2009). Reading habits for both words and numbers contribute to the SNARC effect. Psychonomic Bulletin and Review, $16(2), 328-331$.

Siegenthaler, E., Costela, F.M., McCamy, M.B., Di Stasi, L.L., Otero-Millan, J., Sonderegger, A., Groner, R., Macknik, S., \& Martinez-Conde, S. (2014). Task difficulty in mental arithmetic affects microsaccadic rates and magnitudes. European Journal of Neuroscience, 39(1), 1-8.

Song, J. H., \& Nakayama, K. (2008). Numeric comparison in a visually guided manual reaching task. Cognition, 106(2), 994-1003.

Spivey, M.J., Grosjean, M., \& Knoblich, G. (2005). Continuous attraction toward phonological competitors. Proceedings of National Academy of Science, 102, $10393-10398$.

Stoianov, I., Kramer, P., Umilta, P., \& Zorzi, M. (2008). Visuospatial priming of the mental number line. Cognition, 106, 770-779. 
Towse, J.N., Loetscher, T., \& Brugger, P. (2014). Not all numbers are equal: Preferences and biases among children and adults when generating random sequences. Frontiers in Psychology, 5, 19.

Trick, L.M. \& Pylyshyn, Z.W. (1994). Why are small and large numbers enumerated differently? A limited-capacity preattentive stage in vision. Psychological Review, 101(1), 80-102.

Tschentscher, N., Hauk, O., Fischer, M., H., \& Pulvermüller, F. (2011). You can count on the motor cortex: Finger counting habits modulate motor cortex activation evoked by numbers. Neuroimage, 59, 3139-3148.

Vainio, L., Ellis, R., \& Tucker, M. (2007). The role of visual attention in action priming. The Quarterly Journal of Experimental Psychology, 60(2), 241-261.

Viarouge, A., Hubbard, E.M., \& Dehaene, S. (2014). The organization of spatial reference frames involved in the SNARC effect. The Quarterly Journal of Experimental Psychology, 67(8), 1484-1499.

Winter, B., Marghetis, T., \& Matlock, T. (2015). Of magnitudes and metaphors: Explaining cognitive interactions between space, time, and number. Cortex, 64, 209-224.

Wood, G., Nuerk, H.-C., Willmes, K., \& Fischer, M. H. (2008). On the cognitive link between space and number: A meta-analysis of the SNARC effect. Psychology Science Quarterly, 50(4), 489-525.

Wurtz, R. H., \& Munoz, D. P. (1995). Role of monkey superior colicullus in control of saccades and fixation. In M. S. Gazzaniga (Ed.), The cognitive neurosciences (pp. 533-548). Cambridge, MA: MIT Press.

Yarbus, A.L. (1967). Eye Movements and Vision. Plenum Press: New York. 
Zwaan, R.A., \& Taylor, L.J. (2006). Seeing, acting, understanding: motor resonance in language comprehension. Journal of Experimental Psychology: General, 135, 111. 